IP $\rightleftharpoons$ B

\title{
Seleção de espécie, temperatura e tempo de carbonização na produção de carvão vegetal com resíduos madeireiros da Amazônia
}

Luan Felipe Feitosa da Silva ${ }^{(D *}{ }^{*}$, Antonio Renan Sales de Castro ${ }^{1}$ (D), Rudson Silva Oliveira ${ }^{1}$ (D), Simonne Sampaio da Silva ${ }^{1+}$ (D), Vanessa Mayara Souza Pamplona ${ }^{1}$ (D), Denes de Souza Barros ${ }^{1}$ (D), João Rodrigo Coimbra Nobre ${ }^{2}$ (D) Sueo Numazawa1 ${ }^{1}$ )

${ }^{1}$ Universidade Federal Rural da Amazônia, Avenida Presidente Tancredo Neves, $n^{\circ} 2501$, Terra Firme, CEP 66077-830, Belém, PA, Brasil

Universidade do Estado do Pará, Rodovia PA-125, s/n, Angelim, CEP 68625-000, Paragominas, PA, Brasil

†In memoriam

"Autor correspondente:

luannsilva05@gmail.com

Termos para indexação:

Resíduos de madeira

Rendimento

Carvão vegetal

Index terms:

Wood waste

Yield

Charcoal

Histórico do artigo:

Recebido em 27/09/2018

Aprovado em 02/03/2020

Publicado em 30/06/2020
Resumo - A atividade florestal na Amazônia gera grandes quantidades de resíduos, desde a exploração florestal até o beneficiamento das toras, sendo a carbonização uma alternativa potencial para mitigar este passivo. Entretanto, a diversidade de espécies e a carência de informações sobre parâmetros ideais de carbonização dificultam a operação dos fornos. Nessa pesquisa são avaliados os efeitos da espécie e parâmetros de carbonização sobre a qualidade energética na conversão de resíduos de indústria madeireira em carvão vegetal. O material de estudo foi proveniente do município de Paragominas, PA, coletando-se amostras das três espécies mais comercializadas, Handroanthus serratifolius (Vahl) S.O., Hymenolobium petraeum Ducke e Enterolobium schomburgkii (Benth.) Benth. As carbonizações foram realizadas em forno mufla, com temperaturas de $500{ }^{\circ} \mathrm{C}$ e $600{ }^{\circ} \mathrm{C}$ e patamar final de 30 min e 60 min. Os parâmetros espécie e temperatura foram os que apresentaram efeito significativo, exercendo maior influência na produção e na qualidade do carvão vegetal. O tempo no patamar final de carbonização não influenciou as variáveis. $\mathrm{O}$ aumento da temperatura provocou redução no rendimento gravimétrico. Os resíduos madeireiros das três espécies apresentaram características de elevada qualidade para produção de carvão vegetal.

\section{Selection of species and carbonization parameters of waste wood in Amazon}

\begin{abstract}
Forestry activities in the Amazon generate large amounts of waste, from logging process, with carbonization being a potential alternative to mitigate this liability. However, the diversity of species and the lack of information on ideal carbonization parameters make the operation of the ovens difficult. In this research, the effects of the species and carbonization parameters on energy quality in the conversion of wood industry residues into charcoal are evaluated. The study material came from the municipality of Paragominas, Pará, collecting samples of the three most commercialized species, Handroanthus serratifolius (Vahl) S.O., Hymenolobium petraeum Ducke and Enterolobium schomburgkii (Benth.) Benth. The carbonizations were carried out in a muffle furnace, with temperatures of $500{ }^{\circ} \mathrm{C}$ and $600{ }^{\circ} \mathrm{C}$ and a final level of $30 \mathrm{~min}$ and $60 \mathrm{~min}$. The species and temperature parameters were those that had a significant effect, exerting the greatest influence on the production and quality of charcoal. The time at the final level of carbonization did not show any influence on the variables. The increase in temperature caused a reduction in gravimetric yield. The wood residues of the three species showed high quality characteristics for the production of charcoal.
\end{abstract}




\section{Introdução}

O desenvolvimento econômico e o progresso da sociedade trouxeram consigo implicações acerca dos recursos energéticos, principalmente, quanto à sua disponibilidade, fonte e eficiência. Assim, surgiu a necessidade de se buscar novas fontes renováveis e o desenvolvimento de tecnologias mais eficientes para produção de energia, no intuito de substituir o uso dos combustíveis fósseis (Feuerbacher et al., 2016; Eloy et al., 2018).

Segundo dados do Balanço Energético Nacional, a participação de fontes renováveis na matriz energética brasileira atingiu 45,3\% em 2018, sendo dividida em utilização de bagaço de cana-de-açúcar $(17,4 \%)$, hidráulica (12,6\%), lenha e carvão vegetal $(8,4 \%)$ e lixiviação e outras fontes (6,9\%) (Brasil, 2019).

A biomassa florestal utilizada para produção de energia é proveniente de plantios florestais para energia, árvores mortas por perturbações, resíduos da colheita (casca, galhos e folhas), desbastes, podas e do processamento industrial (Ferreira et al., 2018). A Lei da Política Nacional dos Resíduos Sólidos, n. ${ }^{\circ}$ 12.305/2010 (Brasil, 2010), prevê o incentivo ao desenvolvimento de sistemas de gestão ambiental e empresarial voltados para a melhoria dos processos produtivos e ao reaproveitamento dos resíduos sólidos, com a recuperação e o aproveitamento energético, fortalecendo a implementação do conceito de economia circular e bioeconomia.

No Brasil, a principal fonte de madeira para a produção de carvão vegetal era proveniente de florestas nativas. Porém, com o aumento das exigências impostas pelas legislações ambientais e a preocupação com a sustentabilidade dos processos de produção de energia a partir da biomassa, as florestas plantadas e os resíduos da indústria da base florestal passaram a ser as principais fontes de matéria prima. Isso contribui com o aumento da renda, agrega valor a produtos e colabora para a conservação da biodiversidade (Jesus et al., 2017; Hilling et al., 2018).

A atividade florestal na Amazônia gera grandes quantidades de resíduos, principalmente durante a colheita (9-18\%)enoprocessode beneficiamentodas toras (45-55\%) (Numazawa et al., 2017).

Em 2010, cerca de 1,6 milhão de $\mathrm{m}^{3}$ de resíduos do processamento mecânico das toras foi destinado à produção de carvão vegetal e outros 2,7 milhões de $\mathrm{m}^{3}$ para a geração direta de energia (Imazon et al. 2010).De acordo com a Empresa de Pesquisa Energética (Brasil, 2018) a Região Amazônica apresenta potencial de utilização dos resíduos do manejo florestal sustentável e do processamento industrial da madeira para abastecer sistemas de produção de carvão vegetal e uso como fonte primária de produção de energia via combustão direta.

No entanto, a tecnologia utilizada para a produção de carvão vegetal na região Norte do Brasil é considerada obsoleta. Em geral, são utilizados fornos de alvenaria onde não se permite o controle adequado dos parâmetros do processo, elevando a dificuldade para se obter produtos com qualidade homogênea (Oliveira et al., 2019). Além disso, o fator espécie, temperatura e tempo final de carbonização são importantes, pois interferem diretamente na qualidade do carvão vegetal, já que diferentes produtos são gerados como função das diferentes reações causadas por essas variáveis (Trugilho \& Silva, 2001; Amutio et al., 2012; Silva et al., 2018b).

Portanto, conhecer e entender a interação entre esses fatores é uma lacuna que necessita ser preenchida para tornar mais eficiente o aproveitamento energético dos resíduos gerados na Amazônia.

Neste contexto, este trabalho teve como objetivo avaliar os efeitos de espécies, de temperaturas e de tempos finais no patamar de carbonização na produção de carvão vegetal com resíduos madeireiros no Estado do Pará.

\section{Material e métodos}

A coleta do material foi realizada em uma serraria, localizada na BR-010, no município de Paragominas, Pará. Entre as coordenadas geográficas $3^{\circ} 30^{\prime} \mathrm{S}$ e $3^{\circ} 45^{\prime}$ $\mathrm{S}$ e $48^{\circ} 30^{\prime} \mathrm{W}$ e $48^{\circ} 45^{\prime} \mathrm{W}$.

Foram coletados resíduos de madeira de Handroanthus serratifolius (Vahl) S.O. (ipê-amarelo), Hymenolobium petraeum Ducke (angelim-pedra), e Enterolobium schomburgkii (Benth.) Benth (orelha-de-macaco), que são as três espécies mais comercializadas pela empresa. Para efeito de comprovação científica das espécies, os resíduos foram encaminhados para o Laboratório de Botânica-Xiloteca da Embrapa Amazônia Oriental, Belém, PA, para a identificação anatômica de cada espécie (laudo NIDX 01/2017).

Em seguida, todo material coletado foi enviado ao Laboratório de Tecnologia e Produtos Florestais (LTPF) da Universidade Federal Rural da Amazônia, 
para produção e determinação da densidade básica da madeira e das propriedades energéticas do carvão vegetal (Tabela 1).

Tabela 1. Procedimentos e normas utilizados para a determinação da densidade básica da madeira e das propriedades energéticas do carvão vegetal.

Table 1. Procedures and standards used for the determination of the basic density of wood and the charcoal energy properties.

\begin{tabular}{cc}
\hline Propriedade & Norma \\
\hline Densidade básica da madeira & NBR 11941 (ABNT, 2003) \\
Densidade básica do carvão & Vital (1984) \\
Rendimento gravimétrico do carvão & Oliveira et al. (2010) \\
Química imediata & D1762-84 (ASTM, 2007) \\
Poder calorífico superior & DE711-87 (ASTM, 2004) \\
\hline
\end{tabular}

Foram preparados 20 corpos de provas com $2 \mathrm{~cm} \mathrm{x}$ $3 \mathrm{~cm}$ x $5 \mathrm{~cm}$ (largura x espessura x comprimento) para avaliação da produção em carvão vegetal, sendo usados 5 corpos de provas para cada carbonização. Os corpos de prova foram identificados, secos em estufa a $105^{\circ} \mathrm{C}$, em seguida envolvidos em papel alumínio e colocados em forno mufla, seguindo a metodologia utilizada por Brand et al. (2013).

Os parâmetros de carbonização utilizados foram: taxa de aquecimento de $1,67^{\circ} \mathrm{C} \mathrm{min}-1$; temperaturas finais de carbonização de $500{ }^{\circ} \mathrm{C}$ e $600{ }^{\circ} \mathrm{C}$; e tempos no patamar final de carbonização de 30 min e $60 \mathrm{~min}$.

$\mathrm{O}$ experimento foi realizado em delineamento inteiramente casualizado (DIC), com arranjo fatorial $3 \times 2 \times 2$ (espécies $\times$ temperatura $\times$ tempo), resultando em 12 tratamentos (Tabela 2), com cinco repetições para o rendimento gravimétrico (RGC) e densidade básica do carvão $(\mathrm{DCv})$; e para o teor de materiais voláteis (TMV), teor de cinzas (TCZ), teor de carbono fixo (TCF) e poder calorífico superior (PCS) foram utilizadas duas repetições. Para a densidade básica da madeira (DBM) os dados foram analisados em DIC simples com 3 tratamentos (espécies) e 20 repetições.

Para verificar o efeito das espécies, temperatura e tempo sobre os parâmetros avaliados, os dados foram submetidos ao teste estatístico de Fisher por meio da análise de variância (ANOVA) a $95 \%$ de probabilidade $(p<0,05)$, inicialmente aplicou-se o teste de Levene, para testar a homogeneidade de variância, e o teste de
Shapiro-Wilk, para testar a normalidade dos dados. Quando identificadas diferenças significativas na ANOVA, aplicou-se o teste de Tukey ao nível de 5\% de significância $(p<0,05)$.

Tabela 2. Marcha de carbonização utilizada no experimento.

Table 2. Carbonization run used in the experiment.

\begin{tabular}{ccc}
\hline Espécies & $\begin{array}{c}\text { Temperatura final de } \\
\text { carbonização }\left({ }^{\circ} \mathbf{C}\right)\end{array}$ & $\begin{array}{c}\text { Tempo no } \\
\text { patamar final de } \\
\text { carbonização } \\
\text { (min) }\end{array}$ \\
\hline \multirow{2}{*}{$\begin{array}{c}\text { Handroanthus } \\
\text { serratifolius }\end{array}$} & 500 & 30 \\
\cline { 2 - 3 } & 600 & 60 \\
\hline Hymenolobium & 500 & 30 \\
petraeum & 600 & 60 \\
\cline { 2 - 3 } & & 30 \\
& 500 & 60 \\
\hline Enterolobium & 600 & 30 \\
schomburgkii & & 60 \\
\cline { 2 - 3 } & & 30 \\
\hline
\end{tabular}

Procedeu-se a análise de correlação linear de Pearson entre as características mensuradas no carvão vegetal, considerando todas as repetições para cada espécie. Posteriormente, com base nas correlações significativas encontradas, foram ajustados modelos de regressão múltipla, conforme a Equação 1.

$$
\mathrm{PCS}=\beta_{0}+\beta_{1} \times \mathrm{TMV}+\beta_{2} \times \mathrm{TCF}+\mathrm{e}
$$

Em que: PCS $=$ valor estimado para o poder calorífico superior $\left(\mathrm{kcal} \mathrm{kg}^{-1}\right) ; \beta_{0}, \beta_{1}$ e $\beta_{2}=$ coeficientes de regressão; $\mathrm{TMV}$ e TCF $=$ teores de materiais voláteis $(\%)$ e de carbono fixo (\%), respectivamente; e = erro de estimativa.

Os cálculos estatísticos foram realizados com o auxílio dos softwares SPSS, versão 20.0 e AgroEstat (Barbosa \& Maldonado Júnior, 2015).

\section{Resultados}

O resultado da análise estatística apontou que há diferença entre as espécies para a densidade básica da madeira (DBM). A análise de variância revelou não haver efeito significativo para a interação entre os tratamentos espécie, temperatura e tempo para a variável densidade básica do carvão (DCv), indicando que os fatores são 
independentes entre si. Contudo, foram observadas diferenças significativas para as interações espécies $\mathrm{x}$ temperatura, para o rendimento gravimétrico (RGC), espécies x tempo, para os teores de materiais voláteis (TMV), de cinzas (TCZ) e de carbono fixo (TCF) e para o poder calorífico superior (PCS).

Tabela 3. Valores do teste de Fisher obtidos com base na análise de variância para a densidade básica da madeira e as propriedades energéticas do carvão vegetal de três espécies da floresta Amazônica.

Table 3. Fisher's test values obtained based on the analysis of variance for the basic density of wood and the charcoal energy properties of three species from Amazon forest.

\begin{tabular}{|c|c|c|c|c|c|c|c|c|}
\hline Fonte de Variação & GL & DBM & $\mathrm{DC}_{\mathrm{v}}$ & RGC & TMV & TCZ & TCF & PCS \\
\hline Espécies (E) & 2 & $1707^{*}$ & $353,63 * *$ & 2,57 & $43,10 * *$ & $8,54 * *$ & $37,24 * *$ & $124,83 * *$ \\
\hline Temperatura (T) & 1 & - & $6,76^{*}$ & $38,27 * *$ & $603,30 * *$ & 0,23 & $548,48 * *$ & $759,11 * *$ \\
\hline Tempo (TE) & 1 & - & 0,01 & 0,51 & 0,03 & 0,02 & 0,02 & $49,80^{* *}$ \\
\hline Interação ExT & 2 & - & 0,73 & $3,45^{*}$ & 1,19 & 0,27 & 0,94 & 3,62 \\
\hline Interação ExTE & 2 & - & 0,15 & 0,55 & $6,39 *$ & 0,27 & $5,42 *$ & 2,81 \\
\hline Interação TxTE & 1 & - & 2,45 & 0,03 & 0,73 & 3,40 & 0,29 & $44,50 * *$ \\
\hline Interação ExTxTE & 2 & - & 0,98 & 0,57 & 0,84 & 2,59 & 1,23 & 1,60 \\
\hline $\mathrm{CVe} \%$ & - & 2,02 & 3,55 & 4,83 & 2,99 & 9,12 & 0,65 & 0,28 \\
\hline
\end{tabular}

$\overline{\mathrm{DBM}}$ = densidade básica da madeira; $\mathrm{DCv}=$ densidade básica do carvão; $\mathrm{RGC}=$ rendimento gravimétrico; $\mathrm{TMV}=$ teor de materiais voláteis; $\mathrm{TCZ}=$ teor de cinzas; TCF = teor de carbono fixo; PCS = poder calorífico superior; $\mathrm{CVe}=$ coeficiente de variação experimental. * e ** Significativo ao nível de $5 \%$ e $1 \%$ de probabilidade, respectivamente.

Foi possível observar que o DCv seguiu a mesma tendência verificada para a DMB. As espécies com maior densidade originaram carvões mais densos, com destaque para H. serratifolius $\left(0,625 \mathrm{~g} \cdot \mathrm{cm}^{-3}\right)$ (Figura 1$)$.

Na Tabela 4 são apresentados os resultados isolados para os fatores espécies, temperatura e tempo final no patamar de carbonização. Verificou-se que o RGC não foi influenciado pelo fator espécie e tempo no patamar final de carbonização. A temperatura de carbonização foi o parâmetro que exerceu maior influência, em que o carvão produzido a $500^{\circ} \mathrm{C}$ foi o que apresentou estatisticamente a maior média.

As espécies que apresentaram os menores TMV foram $H$. serratifolius e $E$. shomburgkii, ambas diferiram estatisticamente de $H$. petraeum. O TMV encontrado para o carvão produzido a $600{ }^{\circ} \mathrm{C}$ foi cerca de $5 \%$ menor quando comparado aquele produzido a $500{ }^{\circ} \mathrm{C}$, sendo estes não influenciados pelo fator tempo no patamar final de carbonização (Tabela 4).
A variável carbono fixo apresentou diferença significativa em todos os fatores avaliados, exceto para tempo de carbonização. $H$. serratifolius apresentou a maior média, seguida de E. shomburgkii e de H. petraeum. A elevação da temperatura final de carbonização de 500 ${ }^{\circ} \mathrm{C}$ para $600{ }^{\circ} \mathrm{C}$ ocasionou aumento de $6,4 \%$ no TCF.

Ainda na Tabela 4 são apresentados os valores médios referentes ao PCS, para as três espécies em estudo, nas duas temperaturas de carbonização testadas, e os tempos finais no patamar de carbonização de 30 e 60 min. Todos os fatores apresentaram diferenças significativas. A espécie que apresentou maior média foi H. serratifolius $\left(7.510 \mathrm{kcal} \mathrm{kg}^{-1}\right)$ e a menor foi H. petraeum (7.340 kcal kg-1), sendo que o carvão vegetal produzido na temperatura final de $600{ }^{\circ} \mathrm{C}$ apresentou o maior poder calorífico $\left(7.540 \mathrm{kcal} \mathrm{kg}^{-1}\right)$.

A variável rendimento gravimétrico do carvão (RGC) mostrou diferença significativa apenas para a interação entre espécie e temperatura, indicando que ambos os fatores não são independentes (Tabela 5). 
Seleção de espécie, temperatura e tempo de carbonização na produção de carvão vegetal com resíduos madeireiros da 5 de 11 Amazônia

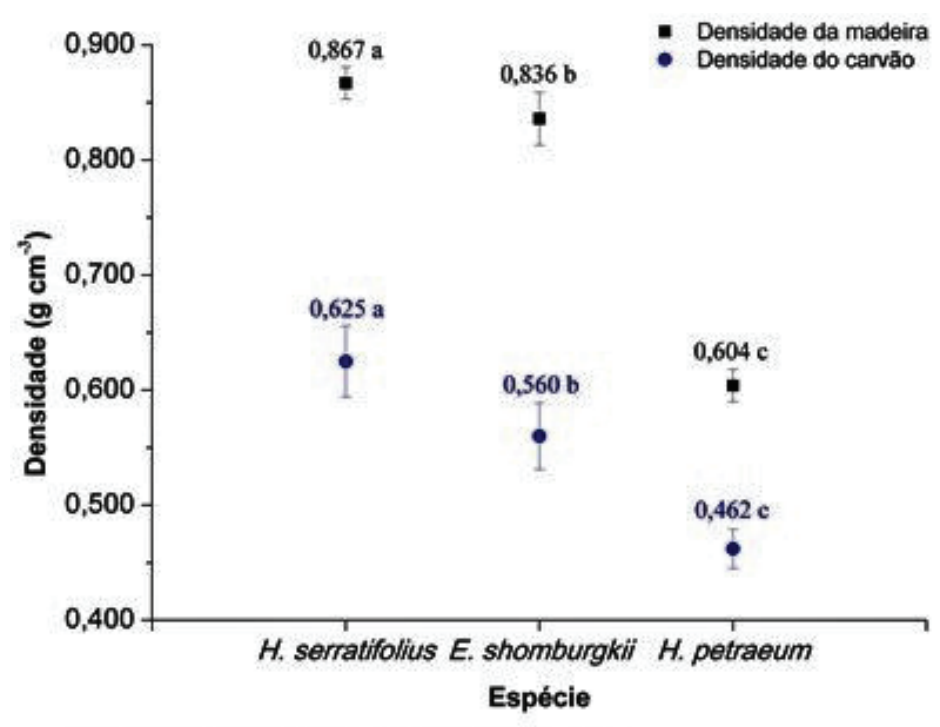

Figura 1. Valores médios para densidade básica da madeira e do carvão vegetal $\left(\mathrm{g} . \mathrm{cm}^{-3}\right)$ Handroanthus serratifolius, Hymenolobium petraeum e Enterolobium schomburgkii da floresta Amazônica. Barras representam o desvio de padrão.

Figure 1. Average values of basic wood density and charcoal $\left(\mathrm{g} . \mathrm{cm}^{-3}\right)$ of Handroanthus serratifolius, Hymenolobium petraeum e Enterolobium schomburgkii from Amazon forest. Bars represent standard deviation.

Tabela 4. Valores médios e desvio-padrão para as propriedades energéticas do carvão vegetal de três espécies da floresta Amazônica.

Table 4. Average values and standard deviation for the charcoal energy properties of three species from Amazon forest.

\begin{tabular}{|c|c|c|c|c|c|}
\hline Parâmetros & $\begin{array}{c}\text { RGC } \\
(\%)\end{array}$ & $\begin{array}{l}\text { TMV } \\
(\%)\end{array}$ & $\begin{array}{l}\text { TCZ } \\
(\%)\end{array}$ & $\begin{array}{l}\text { TCF } \\
(\%)\end{array}$ & $\begin{array}{c}\text { PCS } \\
\left(\mathrm{kcal} \mathrm{kg}^{-1}\right)\end{array}$ \\
\hline \multicolumn{6}{|c|}{ Espécie } \\
\hline Handroanthus serratifolius & $33,53 \pm 0,01 \mathrm{a}$ & $16,04 \pm 3,02 b$ & $0,84 \pm 0,04 \mathrm{~b}$ & $83,11 \pm 3,01 \mathrm{a}$ & $7.510 \pm 0,15 \mathrm{a}$ \\
\hline Enterolobium schomburgkii & $32,59 \pm 0,03 \mathrm{a}$ & $16,63 \pm 2,77 b$ & $0,98 \pm 0,13 \mathrm{a}$ & $82,39 \pm 0,99 \mathrm{~b}$ & $7.420 \pm 0,11 b$ \\
\hline Hymenolobium petraeum & $32,49 \pm 0,02 \mathrm{a}$ & $18,32 \pm 2,57 \mathrm{a}$ & $0,83 \pm 0,05 \mathrm{~b}$ & $80,85 \pm 2,59 \mathrm{c}$ & $7.340 \pm 0,14 \mathrm{c}$ \\
\hline \multicolumn{6}{|c|}{ Temperatura } \\
\hline $500^{\circ} \mathrm{C}$ & $34,14 \pm 0,01 \mathrm{a}$ & $19,55 \pm 1,09 \mathrm{a}$ & $0,89 \pm 0,09 \mathrm{a}$ & $79,56 \pm 1,09 \mathrm{~b}$ & $7.300 \pm 0,10 b$ \\
\hline $600^{\circ} \mathrm{C}$ & $31,61 \pm 0,02 \mathrm{~b}$ & $14,45 \pm 1,24 \mathrm{~b}$ & $0,88 \pm 1,24 \mathrm{a}$ & $84,68 \pm 1,21 \mathrm{a}$ & $7.540 \pm 0,07 \mathrm{a}$ \\
\hline \multicolumn{6}{|c|}{ Tempo } \\
\hline $30 \mathrm{~min}$ & $33,02 \pm 0,03 \mathrm{a}$ & $17,01 \pm 2,99 \mathrm{a}$ & $0,88 \pm 0,11 \mathrm{a}$ & $82,10 \pm 2,96 \mathrm{a}$ & $7.390 \pm 0,17 b$ \\
\hline $60 \mathrm{~min}$ & $32,73 \pm 0,02 \mathrm{a}$ & $16,98 \pm 2,82 \mathrm{a}$ & $0,89 \pm 0,11 \mathrm{a}$ & $82,13 \pm 2,86 \mathrm{a}$ & $7.450 \pm 0,13 \mathrm{a}$ \\
\hline
\end{tabular}

$\overline{\mathrm{RGC}}=$ rendimento gravimétrico; TMV $=$ teor de materiais voláteis; $\mathrm{TCZ}=$ teor de cinzas; $\mathrm{TCF}=$ teor de carbono fixo; $\mathrm{PCS}=$ poder calorífico superior. Médias seguidas de mesma letra, na coluna para cada parâmetro avaliado, não diferem entre si, pelo teste de Tukey $(p<0,05)$. 
Tabela 5. Desdobramento da interação entre espécies e temperatura para rendimento gravimétrico do carvão (RGC) de três espécies da floresta Amazônica.

Table 5. Deployment of interaction between species and temperatures for charcoal gravimetric yield (RGC) of three species from Amazon forest.

\begin{tabular}{|c|c|c|c|c|}
\hline \multirow{3}{*}{ Espécie } & \multicolumn{4}{|c|}{ Temperatura } \\
\hline & $500^{\circ} \mathrm{C}$ & & $600^{\circ} \mathrm{C}$ & \\
\hline & \multicolumn{4}{|c|}{ RGC (\%) } \\
\hline Handroanthus serratifolius & $34,04 \pm 0,002$ & $\mathrm{aA}$ & $33,01 \pm 0,01$ & $\mathrm{aA}$ \\
\hline Enterolobium shomburgkii & $34,38 \pm 0,002$ & $\mathrm{aA}$ & $30,81 \pm 0,03$ & $\mathrm{bB}$ \\
\hline Hymenolobium petraeum & $34,00 \pm 0,004$ & $\mathrm{aA}$ & $31,00 \pm 0,01$ & $\mathrm{bB}$ \\
\hline
\end{tabular}

* Letras iguais minúsculas nas linhas e maiúsculas nas colunas não diferem entre si, pelo teste de Tukey $(\mathrm{p}>0,05)$.

Apenas $H$. serratifolius manteve valor constante para RGC, tanto para carbonização a $600^{\circ} \mathrm{C}$ quanto a $500^{\circ} \mathrm{C}$. Em contra partida, para E. shomburgkii e H. petraeum, o aumento da temperatura de carbonização provocou diminuição no RGC.

Tabela 6. Desdobramento do efeito de espécie e tempo de carbonização para o teor de materiais voláteis (TMV) e teor de carbono fixo (TCF) para três espécies da Floresta Amazônica.

Table 6. Deployment effect of species and time of carbonization for volatile material rate (TMV) and fixed carbon rate (TCF) of three species from Amazon Forest.

\begin{tabular}{|c|c|c|c|c|}
\hline \multirow{3}{*}{ Espécie } & \multicolumn{4}{|c|}{ Tempo } \\
\hline & $30 \mathrm{~min}$ & $60 \mathrm{~min}$ & $30 \mathrm{~min}$ & $60 \mathrm{~min}$ \\
\hline & \multicolumn{2}{|c|}{ TMV (\%) } & \multicolumn{2}{|c|}{ TCF (\%) } \\
\hline Handroanthus serratifolius & $16,49 \pm 3,52 \mathrm{bB}$ & $15,59 \pm 2,89 \mathrm{cB}$ & $82,69 \pm 3,53 \mathrm{aB}$ & $83,54 \pm 2,85 \mathrm{aA}$ \\
\hline Enterolobium shomburgkii & $16,17 \pm 2,93 \mathrm{bB}$ & $17,09 \pm 2,95 \mathrm{bA}$ & $82,84 \pm 2,89 \mathrm{aA}$ & $81,94 \pm 3,06 \mathrm{bB}$ \\
\hline Hymenolobium petraeum & $18,38 \pm 2,83 \mathrm{aA}$ & $18,25 \pm 2,73 \mathrm{aA}$ & $80,79 \pm 2,81 \mathrm{bA}$ & $80,92 \pm 2,78 \mathrm{cA}$ \\
\hline
\end{tabular}

* Letras iguais minúsculas nas linhas e maiúsculas nas colunas não diferem entre si, pelo teste de Tukey $(\mathrm{p}>0,05)$.

Tabela 7. Desdobramento da interação entre a temperatura e o tempo no patamar final de carbonização para o poder calorífico superior (PCS) para três espécies da Floresta Amazônica.

Table 7. Deployment of interaction between temperatures and times at final carbonization level for superior calorific power (PCS) of three species from Amazon Forest.

\begin{tabular}{|c|c|c|}
\hline \multirow{3}{*}{ Temperatura } & \multicolumn{2}{|c|}{ Tempo } \\
\hline & $30 \mathrm{~min}$ & $60 \mathrm{~min}$ \\
\hline & \multicolumn{2}{|c|}{ PCS (kcal kg-1) } \\
\hline $500^{\circ} \mathrm{C}$ & $7250 \pm 0,07 \mathrm{bB}$ & $7360 \pm 0,09 \mathrm{bA}$ \\
\hline $600^{\circ} \mathrm{C}$ & $7540 \pm 0,08 \mathrm{aA}$ & $7540 \pm 0,08 \mathrm{aA}$ \\
\hline
\end{tabular}

* Letras iguais minúsculas nas linhas e maiúsculas nas colunas não diferem entre si, pelo teste de Tukey $(\mathrm{p}>0,05)$.
Houve interação significativa para os fatores espécies e tempo de carbonização para o TMV e TCF (Tabela 6). Houve diminuição dos constituintes químicos no carvão vegetal, responsáveis pela formação dos materiais voláteis durante a reação de carbonização, com maior tempo de exposição ao calor da pirólise. A variação do tempo de carbonização de 30 para 60 min proporcionou um aumento na concentração de carbono fixo em $H$. serratifolius e $H$. petraeum.

Foi observada diferença significativa do PCS $\left(\mathrm{kcal} \mathrm{kg}^{-1}\right)$ entre as temperaturas e tempo no patamar final de carbonização. O teste de comparação de média não detectou diferenças para as carbonizações de 30 e 60 min a $600{ }^{\circ} \mathrm{C}$. Entretanto, a carbonização com patamar de $500{ }^{\circ} \mathrm{C}$ e também com tempo final de $30 \mathrm{~min}$ apontou diferenças, com destaque para $600{ }^{\circ} \mathrm{C}$ que com essas configurações apresentou a maior média.
As correlações entre as propriedades químicas do carvão vegetal de cada espécie estão apresentadas na Tabela 8. Verificou-se que todas as espécies estudadas apresentaram comportamento semelhante para os resultados das correlações entre as propriedades. $\mathrm{O}$ TMV do carvão apresentou correlação inversamente proporcional e altamente significativa com o TCF e PCS, sendo o TCF correlacionado positivamente com o PCS.

$\mathrm{Na}$ Tabela 9 são apresentadas as equações ajustadas e os seus respectivos coeficientes de determinação $\left(\mathrm{R}^{2}\right)$ obtidos para o PCS em função dos TMV e TCF. O teste $\mathrm{F}$ da análise de variância foi significativo para todas as equações. 
Tabela 8. Matriz com os coeficientes de correlação de Pearson entre as variáveis analisadas para três espécies da Floresta Amazônica.

Table 8. Matrix with the Pearson correlation coefficients between the variables analyzed of three species from Amazon Forest.

\begin{tabular}{cccccc}
\hline Espécie & Variável & TMV & TCZ & TCF & PCS \\
\hline \multirow{4}{*}{$\begin{array}{c}\text { Handroanthus } \\
\text { serratifolius }\end{array}$} & TMV & 1,000 & $-0,279$ & $-1,000^{* *}$ & $-0,966^{* *}$ \\
& TCZ & & 1,000 & 0,266 & 0,280 \\
& TCF & & & 1,000 & $0,965^{* *}$ \\
& PCS & & & & 1,000 \\
\hline \multirow{4}{*}{$\begin{array}{c}\text { Enterolobium } \\
\text { shomburgkii }\end{array}$} & TMV & 1,000 & 0,216 & $-0,999^{* *}$ & $-0,866^{* *}$ \\
& TCZ & & 1,000 & $-0,260^{\text {NS }}$ & 0,003 \\
& TCF & & & 1,000 & $0,856^{* *}$ \\
& PCS & & & & 1,000 \\
\hline \multirow{2}{*}{$\begin{array}{c}\text { Hymenolobium } \\
\text { petraeum }\end{array}$} & TMV & 1,000 & 0,323 & $-1,000^{* *}$ & $-0,941^{* *}$ \\
& TCZ & & 1,000 & $-0,338$ & $-0,177$ \\
& TCF & & & 1,000 & $0,938^{* *}$ \\
& PCS & & & & 1,000 \\
\hline
\end{tabular}

** correlação significativa a $1 \%$ de probabilidade; TMV, TCZ e TCF $=$ teores de materiais voláteis, cinzas e carbono fixo, respectivamente (\%); PCS = poder calorífico superior do carvão vegetal $\left(\mathrm{kcal} \mathrm{kg}^{-1}\right)$.

Tabela 9. Equações de regressão ajustadas a partir das propriedades químicas do carvão de três espécies da Floresta Amazônica.

Table 9. Regression equations adjusted from the charcoal chemical properties of three species from Amazon Forest.

\begin{tabular}{ccccc}
\hline Espécie & Equação ajustada & $\mathbf{R}^{2}$ & $\mathbf{F}$ & $\mathbf{p}_{\text {valor }}$ \\
\hline $\begin{array}{c}\text { Handroanthus } \\
\text { serratifolius }\end{array}$ & $\mathrm{PCS}=12,44-0,09 \mathrm{TMV}-0,04 \mathrm{TCF}$ & 0,93 & 34,57 & 0,001 \\
$\begin{array}{c}\text { Enterolobium } \\
\text { shomburgkii }\end{array}$ & $\mathrm{PCS}=25,51-0,21 \mathrm{TMV}-0,18 \mathrm{TCF}$ & 0,78 & 9,27 & 0,021 \\
$\begin{array}{c}\text { Hymenolobium } \\
\text { petraeum }\end{array}$ & $\mathrm{PCS}=52,39-0,50 \mathrm{TMV}-0,44 \mathrm{TCF}$ & 0,90 & 23,15 & 0,003 \\
\hline
\end{tabular}

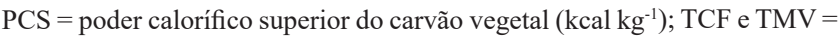
teores de carbono fixo (\%) e de materiais voláteis $(\%)$, respectivamente; $\mathrm{R}^{2}$ = coeficiente de determinação.

\section{Discussão}

Os valores médios de densidade básica da madeira (DBM) (Figura 1), foram próximos ao encontrado por Silva et al. (2018a), que observaram para as espécies Handroanthus serratifolius e Hymenolobium petraeum, 0,52 g.cm ${ }^{-3}$ e 0,93 g.cm ${ }^{-3}$, respectivamente, enquanto, Paula \& Costa (2011) registraram $0,87 \mathrm{~g} \cdot \mathrm{cm}^{-3}$ para a madeira de Enterolobium shomburgkii.
De acordo com Csanády et al. (2015), H. serratifolius e E. shomburgkii. podem ser classificadas como madeiras pesadas, enquanto que o $H$. petraeum de média densidade.

A densidade básica pode variar para uma mesma espécie. Segundo Chave et al. (2006) e Hietz et al. (2013) isso pode ocorrer como reflexo de mudanças nas condições ambientais, assim como idade e das características dendrométricas do indivíduo. Quando se trata de Amazônia essa disparidade aumenta. A procedência das madeiras muitas vezes é desconhecida, em relação às características especificas do ambiente. A variabilidade na densidade pode ser uma das grandes responsáveis pela não homogeneidade do carvão vegetal produzido na região, além da heterogeneidade da madeira das diferentes espécies utilizadas para esse fim.

Apesar das diferenças de densidade entre as três espécies estudadas $\left(0,60\right.$ g.cm ${ }^{-3}$ a 0,87 g.cm $\left.{ }^{-3}\right)$, os resíduos provenientes de todos os indivíduos apresentam potencial para a produção de carvão vegetal. O carvão vegetal produzido a partir desses materiais apresenta maiores densidades básica e energética, devido ao elevado percentual de lignina encontrado em madeiras tropicais, conforme relatado por Couto et al. (2015).

A densidade da madeira é importante para a produção de carvão vegetal, considerando que essa tem relação direta com a densidade do carvão, pois quanto maior for a densidade do carvão, maior será a quantidade de energia liberada por unidade de volume (Silva et al., 2007). Correlação positiva entre a densidade básica da madeira com a densidade do carvão vegetal também foi observada por Brito \& Barrichelo (1977) e Pereira et al. (2016) ao estudarem diferentes materiais genético do gênero Eucalyptus. Em geral, essa característica resulta em aumento da resistência mecânica do carvão, fato importante, especialmente quando o uso do produto for para fins siderúrgicos (Santos et al. 2011).

De acordo com Vale et al. (2010), a densidade básica do carvão deve ser a maior possível, para que apresente alta resistência mecânica e elevada densidade energética por unidade de volume, refletindo em menores custos relacionados ao transporte. Segundo Silva et al. (2007), a relação direta entre a densidade básica da madeira com a densidade básica do carvão intensifica a importância da separação de resíduos por espécies, assim como a sua escolha para produção de carvão vegetal.

A redução no rendimento gravimétrico do carvão, observada a $600{ }^{\circ} \mathrm{C}$, ocorreu devido à maior perda de 
massa resultante da degradação térmica dos constituintes do lenho a partir do incremento da temperatura final de carbonização. Outros autores, como Vieira et al. (2013) e Siebeneichler et al. (2017), ao estudarem carvão vegetal de Eucalyptus spp., espécies muito utilizadas como matéria-prima para carvão vegetal, verificaram diminuição do rendimento em carvão com o aumento de temperatura. Moulin et al. (2017) carbonizaram resíduos de madeira de ipê, em temperaturas de 500 a $700{ }^{\circ} \mathrm{C}$. Esses autores também constataram diminuição no rendimento gravimétrico do carvão com o aumento da temperatura.

Boas et al. (2010) constataram que com o aumento da temperatura de carbonização houve decomposição dos constituintes químicos das matérias-primas, provocando perda de massa e, consequentemente, redução no rendimento gravimétrico do carvão. Vale et al. (2010), ao estudarem espécies nativas brasileiras empregadas para atividade de carvoejamento, observaram comportamento semelhante ao encontrado para espécies do gênero Eucalyptus (31\% a 35\%) utilizados na produção de carvão vegetal para siderurgia.

O aumento do tempo no patamar final de carbonização implica em redução no teor de materiais voláteis (Tabela 6). Enquanto o carvão vegetal residir dentro do forno, este estará sob degradação, refletindo também no rendimento gravimétrico e no teor de carbono fixo.

Ao carbonizar madeiras de espécies amazônicas em escala industrial, em diferentes tempos de carbonização, Silva et al. (2007) verificaram redução no conteúdo de voláteis, com o aumento no tempo de exposição do carvão, durante o processo.

Teores de materiais voláteis semelhantes ou superiores aos encontrados nesse trabalho, para as três espécies da Amazônia, foram relatados por Soares et al. (2015) para o carvão vegetal produzido a partir do clone Eucalyptus grandis $x$ Eucalyptus urophylla $(18,80 \%$ a $22,82 \%)$ e por Neves et al. (2011), que estudaram seis clones de Eucalyptus grandis $x$ Eucalyptus camaldulensis $(18,94 \%)$.

Segundo Brand (2010), baixos teores de materiais voláteis tendem a altos teores de carbono fixo no carvão vegetal, ou seja, eles poderão requerer longo tempo de residência na fornalha para queima total. Contudo, o carvão com elevado conteúdo de voláteis pode ser de fácil inflamabilidade, por necessitar de baixas temperaturas de ignição, desde que a o sistema de conversão esteja dimensionado para fornecer a quantidade de ar necessária para que ocorra um regime de combustão completa, além da granulometria das peças, que exerce forte influência na combustibilidade, temperatura máxima fornecida e tempo de residência (Dias Júnior et al., 2015).

O teor de carbono fixo apresenta um comportamento inversamente proporcional ao conteúdo de materiais voláteis e cinzas, justificando as baixas médias para voláteis $(14,76 \%)$ e o elevado carbono fixo $(84,68 \%)$ na maior temperatura de carbonização $\left(600{ }^{\circ} \mathrm{C}\right)$. Esta variável é o componente predominante do carvão vegetal. Portanto, quanto maior o seu valor maior será a energia de combustão e o seu valor calórico, além de apresentar um regime de queima mais lento, podendo residir por mais tempo no aparelho destinado à queima (Brand, 2010).

Essas duas propriedades se comportam de forma inversa durante o processo de degradação térmica, pois quanto maior a exposição ao calor, mais elementos gasosos são desprendidos da madeira e, consequentemente, há maior concentração da fração sólida (carbono fixo) (Silva et al. 2007).

Ao estudarem o carvão vegetal produzido a $450{ }^{\circ} \mathrm{C}$, utilizando madeira de cedrinho (Scleronema sp.) e de louro (Ocotea sp.), ambas espécies amazônicas, Silva et al. (2014) encontraram $69,54 \%$ e $71,73 \%$ de carbono fixo, respectivamente. Esses baixos valores podem ter sido decorrente da baixa temperatura de carbonização e pela diferente constituição química de cada espécie.

O carvão vegetal que possui elevadas concentrações de carbono fixo é indicado para uso siderúrgico, pois em geral apresenta menor concentração de materiais voláteis e maior conteúdo de carbono, principal elemento necessário para a redução do ferro durante a produção de gusa. Segundo Brito (1993), as fissuras decorrentes da eliminação dos materiais voláteis durante o aquecimento seriam menores, diminuindo-se assim o índice de redução granulométrica e de geração de finos no alto forno.

A análise de TCZ do carvão vegetal revela a ocorrência de diferença significativa entre as espécies (Tabela 4). No entanto, não foram observadas diferenças significativas em relação às temperaturas e tempos de carbonização. Acredita-se que este resultado pode ser intrínseco à espécie e ao sítio (localidade), pois a definição desta propriedade depende da composição inorgânica presente na madeira (Brand, 2010).

No emprego energético do carvão vegetal, a quantidade de energia calorífica liberada pelo combustível é a 
informação chave para se ajustar a quantidade de carga a ser inserida no sistema, com intuito de atender a uma determinada produtividade energética (Brand, 2010). Logo, os materiais analisados apresentam potencial de uso como fonte primária de energia (Tabela 8).

Ao avaliar carvão vegetal de jatobá (Hymenea courbaril L.) produzido a diferentes temperaturas $\left(300\right.$ a $\left.900{ }^{\circ} \mathrm{C}\right)$, Trugilho \& Silva (2001) atestaram que o aumento de temperatura durante o processo de carbonização ocasiona um incremento considerável no poder calorífico superior, estando associado ao elevado conteúdo de carbono fixo.

Numazawa (1990) obteve resultado semelhante aos deste estudo para poder calorífico superior do carvão vegetal produzido a partir dos resíduos da madeira de espécie nativa da Amazônia (cupiúba, Goupia glabra Aubl.), carbonizados a $600{ }^{\circ} \mathrm{C}$, com valor médio de $7.600 \mathrm{kcal} . \mathrm{kg}^{-1}$. No entanto, valores energéticos mais baixos foram relatados por Soares et al. (2015) e Santos et al. (2016), quando analisaram carvão vegetal proveniente do gênero Eucalyptus (7.193 a 7.478 kcal. $\mathrm{kg}^{-1}$ ), indicando que os combustíveis analisados nesta pesquisa apresentam potencial energético.

Se as variáveis consideradas na produção de carvão fossem somente densidade básica da madeira e densidade do carvão vegetal, a espécie Handroanthus serratifolius seria a mais adequada. Contudo, se considerarmos rendimento gravimétrico do carvão, as três espécies seriam indicadas, por apresentarem valor acima de $30 \%$ de rendimento em massa.

Em relação ao aproveitamento de resíduos da indústria madeireira, as três espécies estudadas podem ser consideradas para a produção de carvão vegetal, pois a carbonização é um sistema alternativo para mitigar o passivo ambiental causado pelos resíduos. Esse uso alternativo dos resíduos evitaria que fossem descartados no meio ambiente, com a vantagem de se obter um produto comercializável de origem renovável e de boa qualidade energética, além de proporcionar aumento da cadeia de valor e maior aproveitamento da biomassa florestal.

\section{Conclusões}

Handroanthus serratifolius, Enterolobium shomburgkii e Hymenolobium petraeum apresentaram potencial para produção de carvão vegetal a partir de seus resíduos industriais. Espécie e temperatura de carbonização são os parâmetros que exercem maior influência sobre o rendimento em carvão vegetal e, também, sobre a qualidade do produto.

A temperatura final de carbonização de $600{ }^{\circ} \mathrm{C}$ provocou redução no rendimento gravimétrico e no teor de materiais voláteis. $\mathrm{O}$ incremento em carbono fixo teve correlação positiva direta para elevação do poder calorífico superior.

O teor de cinzas do carvão vegetal não foi influenciado pelos parâmetros de carbonização, indicando sua utilização para uso doméstico e industrial.

As características qualitativas do carvão de resíduos industriais indicaram ser de elevada qualidade para uso doméstico e também para uso na siderurgia devido aos altos valores de densidade e de carbono fixo.

\section{Referências}

ASTM. American Society for Testing and Materials. ASTM D-176284: standard method for chemical analyses of wood charcoal. Philadelphia, 2007. 2 p.

ASTM. American Society for Testing and Materials. ASTM E-71187: standard test method for gross calorific value of refuse-derived fuel by the bomb calorimeter. Philadelphia, 2004. 8 p.

Amutio, M. et al. Influence of temperature on biomass pyrolysis in a conical spouted bed reactor. Resources, Conservation And Recycling, v. 59, p. 23-31, 2012. https://doi.org/10.1016/j. resconrec.2011.04.002.

ABNT. Associação Brasileira de Normas Técnicas. NBR 11941: determinação da densidade básica em madeira. Rio de Janeiro, 2003. 6 p.

Barbosa, J. C. \& Maldonado Júnior, W. Experimentação agronômica \& agroestat: sistema para análises estatísticas de ensaios agronômicos. Jaboticabal: Multipress, 2015.

Boas, M. A. et al. Efeito da temperatura de carbonização e dos resíduos de macaúba na produção de carvão vegetal. Scientia Forestalis, v. 38, n. 87, p. 481-490, 2010.

Brand, M. A. Análise da qualidade da madeira e do carvão vegetal produzido a partir da espécie Miconia cinnamomifolia (De Candolle) Naudin (Jacatirão-açu) na agricultura familiar, em Biguaçu, Santa Catarina. Scientia Forestalis, v. 41, n. 99, p. 401-10, 2013.

Brand, M. A. Energia de biomassa florestal. Rio de Janeiro: Interciência, 2010.

Brasil. Empresa de Pesquisa Energética. Balanço energético nacional 2019: ano base 2018. 12. ed. Rio de Janeiro, 2019.

Brasil. Empresa de Pesquisa Energética. Potencial energético de resíduos florestais do manejo sustentável e de resíduos da industrialização da madeira. Disponível em: <http:// www.epe.gov.br/sitespt/publicacoesdadosabertos/publicacoes/ PublicacoesArquivos/publicacao-312/NT-EPE 17-2018 Biomassa Lenhosa-Residual_2018-10-17.pdf>.Acesso em: 15 jul. 2019.

Pesq. flor. bras., Colombo, v. 40, e201801737, p. 1-11, 2020 
Brasil. Lei $\mathbf{n}^{\circ} \mathbf{1 2 . 3 0 5}$ de $\mathbf{2}$ de agosto de 2010. Institui a Política Nacional de Resíduos Sólidos; altera a Lei no 9.605, de 12 de fevereiro de 1998; e dá outras providências. Disponível em: <http:// www.planalto.gov.br/ccivil_03/_ato2007-2010/2010/lei/112305. htm>. Acesso em: 10 set. 2019.

Brito, J. O. \& Barrichello, L. E. G. Correlações entre características físicas e químicas da madeira e a produção de carvão: 1 . Densidade básica e teor de lignina da madeira de eucalipto. Instituto de Pesquisa e Estudos Florestais, n. 14, p. 9-20, 1977.

Brito, J. O. Reflexões sobre a qualidade do carvão vegetal para uso siderúrgico. Piracicaba: IPEF, 1993. (Circular técnica, 181).

Chave, J. et al. Regional and phylogenetic variation of wood density across 2,456 neotropical tree species. Ecological Applications, v. 16, n. 6, p. 2356-2367, 2006.

Couto, A. M. et al. Qualidade do carvão vegetal de Eucalyptus e Corymbia produzido em diferentes temperaturas finais de carbonização. Scientia Forestalis, v. 43, n. 108, p. 817-831, 2015. https://doi.org/10.18671/scifor.v43n108.7.

Csanády, E. et al. Quality of machined wood surfaces. [S.1.]: Springer, 2015. https://doi.org/10.1007/978-3-319-22419-0.

Dias Júnior, A. F. et al. Granulometric influence on the combustion of charcoal for barbecue. Revista Árvore, v. 39, n. 6, p. 1127-1133, 2015. https://doi.org/10.1590/0100-67622015000600016.

Eloy, E. et al. Effect of age and spacing on biomass production in forest plantation. Revista Árvore, v. 42, n. 2, 2018. https://doi. org/10.1590/1806-90882018000200014.

Ferreira, L. R. A. et al. Review of the energy potential of the residual biomass for the distributed generation in Brazil. Renewable And Sustainable Energy Reviews, v. 94, p. 440-455, 2018. https://doi. org/10.1016/j.rser.2018.06.034.

Feuerbacher, A. et al. Increasing forest utilization within Bhutan's forest conservation framework: the economic benefits of charcoal production. Forest Policy And Economics, v. 73, p. 99-111, 2016. https://doi.org/10.1016/j.forpol.2016.08.007.

Hietz, P. et al. Strong radial variation in wood density follows a uniform pattern in two $n$ neotropical rain forests. Functional Ecology, v. 27, p. 684-692, 2013.

Hillig, E. et al. Propriedades físico-mecânicas da madeira de uvado-japão. Pesquisa Florestal Brasileira, v. 38, p. 1-6, 2018.https:// doi.org/10.4336/2018.pfb.38e201601195.

Jesus, M. S. et al. Caracterização energética de diferentes espécies de Eucalyptus. Floresta, v. 47, n. 1, p. 11-16, 2017. https://doi. org/10.5380/rf.v47i1.48418.

Moulin, J. C. et al. Effect of extractives and carbonization temperature on energy characteristics of wood waste in amazon rainforest. Cerne, v. 23, n 2, p. 209-218, 2017. https://doi.org/10.1590/01047760201 723022216.

Neves, T. A. et al. Avaliação de clones de Eucalyptus em diferentes locais visando à produção de carvão vegetal. Pesquisa Florestal Brasileira, v. 31, n. 68, p. 319-330, 2011.

Numazawa, S. Características do carvão de Cupiuba (Goupia glabra AUBL). Belém: FACP, 1990. 15 p. (FCAP. Informe técnico, $15)$.
Numazawa, C. T. D. et al. Logging residues and CO2 of Brazilian Amazon timber: two case studies of forest harvesting. Resources, Conservation \& Recycling, v. 122, p. 280-285, 2017. https://doi. org/10.1016/j.resconrec.2017.02.016.

Oliveira, A. C. et al. Parâmetros de qualidade da madeira e do carvão vegetal de Eucalyptus pellita F. Muell. Scientia Forestalis, v. 38, n. 87,2010 .

Oliveira, R. S. et al. Qualidade do carvão vegetal comercializado no Sudeste Paraense para cocção de alimentos. Revista de Ciências Agrárias, v. 62, p. 1-9, 2019. https://doi.org/10.22491/rca.2019.3017.

Paula, L. E. \& Costa, K. P. Densidade de madeira de 932 espécies nativas do Brasil. Porto Alegre: Cinco Continentes, 2011.

Pereira, B. L. C. et al. Efeito da carbonização da madeira na estrutura anatômica e densidade do carvão vegetal de Eucalyptus. Ciência Florestal, v. 26, n. 2, p. 545-557, 2016. https:// doi.org/10.5902/1980509822755.

Santos, R. C. et al. Correlações entre os parâmetros de qualidade da madeira e do carvão vegetal de clones de eucalipto. Scientia Forestalis, v. 39, n. 90, p. 221-230, 2011.

Santos, R. C. et al. Influência das propriedades químicas e da relação siringil/guaiacil da madeira de eucalipto na produção de carvão vegetal. Ciência Florestal, v. 26, n. 2, p. 657-669, 2016. https://doi. org/10.5902/1980509822765.

Siebeneichler, E. A. et al. Influência de temperatura e taxas de aquecimento na resistência mecânica, densidade e rendimento do carvão da madeira de Eucalyptus cloeziana. Revista Ciência da Madeira, v. 8, n. 2, 2017. https://doi.org/10.15210/cmad.v8i2.10511.

Silva, D. A. et al. Avaliação das propriedades energéticas de resíduos de madeiras tropicais com uso da espectroscopia NIR. Floresta e Ambiente, v. 21, n. 4, p. 561-568, 2014.

Silva, L. et al. Aproveitamento energético de resíduos da indústria moveleira. Revista Brasileira de Engenharia e Sustentabilidade, v. 5, n. 2, p. 36-42, 2018a. https://doi.org/10.15210/rbes.v5i2.14557.

Silva, M. G. et al. Carvão de resíduos de indústria madeireira de três espécies florestais exploradas no município de Paragominas, PA. Acta Amazonica, v. 37, n. 1, p. 61-70, 2007. https://doi. org/10.1590/s0044-59672007000100007.

Silva, R. C. et al. Influência da temperatura final de carbonização nas características do carvão vegetal de espécies tropicais. Pesquisa Florestal Brasileira, v. 38, p. 1-10, 2018b. https://doi. org/10.4336/2018.pfb.38e201801573.

Soares, V. C. et al. Análise das propriedades da madeira e do carvão vegetal de híbridos de eucalipto em três idades. Cerne, v. 21, n. 2, p. 191-197, 2015. https://doi.org/10.1590/01047760201521021294.

Trugilho, P. F. \& Silva, D. A. Influência da temperatura final de carbonização nas características físicas e químicas do carvão vegetal de jatobá (Himenea courbaril L.). Scientia Agraria, v. 2, n. 1, p. 45-53, 2001. http://dx.doi.org/10.5380/rsa.v2i1.976.

Vale, A. T. et al. Relação entre as propriedades químicas, físicas e energéticas da madeira de cinco espécies do cerrado. Ciência Florestal, v. 20, n. 1, p. 137-145, 2010. https://doi. org/10.5902/198050981767. 
Seleção de espécie, temperatura e tempo de carbonização na produção de carvão vegetal com resíduos madeireiros da 11 de 11 Amazônia

Vieira, R. S. et al. Influência da temperatura no rendimento dos produtos da carbonização de Eucalyptus microcorys. Cerne, v. 19, n. 1, p. 59-64, 2013.
Vital, B. R. Métodos de determinação de densidade da madeira. Viçosa, MG: SIF, 1984. 21 p. (Boletim técnico, 1). 